\title{
Design and development of handover simulator model in 5G cellular network
}

\author{
Abdulkarem Basil Abdulkarem ${ }^{1}$, Lukman Audah ${ }^{2}$ \\ ${ }^{1,2}$ Wireless and Radio Science Centre (WARAS), Universiti Tun Hussein Onn Malaysia, Batu Pahat, Johor, Malaysia \\ ${ }^{1}$ Department of Computer Engineering Techniques, Al-Maarif University College, Ramadi, Iraq
}

\begin{tabular}{|c|c|}
\hline Article Info & ABSTRACT \\
\hline Article history: & In the modern era of technology, the high speed internet is the most \\
\hline Received Sep 4, 2020 & $\begin{array}{l}\text { important part of human life. The current available network is reckoned to be } \\
\text { slow in speed and not be up to snuff for data transmission regarding business }\end{array}$ \\
\hline Revised Dec 6, 2020 & applications. The objective of handover mechanism is to reassign the current \\
\hline Accepted Jan 19, 2021 & $\begin{array}{l}\text { session handle by internet gadget. The globe needs the next generation high } \\
\text { mobility and throughput performance based internet model. This research }\end{array}$ \\
\hline Keywords: & $\begin{array}{l}\text { paper explains the proposed method of design and development for handover } \\
\text { based } 5 \mathrm{G} \text { cellular network. In comparison to the traditional method, we }\end{array}$ \\
\hline Handover & propose to control the handovers between base-stations using a concentric \\
\hline LTE & $\begin{array}{l}\text { method. The channel simulator is applied over the range of the frequencies } \\
\text { from } 500 \mathrm{MHz} \text { to } 150 \mathrm{GHz} \text { and radio frequency for the } 700 \mathrm{MHz} \text { bandwidth. }\end{array}$ \\
\hline MANET & The performance of the simulation system is calculated on the basis of \\
\hline SDN & handover preparation and completion time regarding base station as well as \\
\hline Simulator & $\begin{array}{l}\text { number of users. From this experiment we achieve the } 7.08 \mathrm{~ms} \text { handover } \\
\text { preparation time and } 9.98 \mathrm{~ms} \text { handover completion time. The author } \\
\text { recommended the minimum handover completion time, perform the high } \\
\text { speed for } 5 \mathrm{G} \text { cellular networks. }\end{array}$ \\
\hline
\end{tabular}

This is an open access article under the CC BY-SA license.

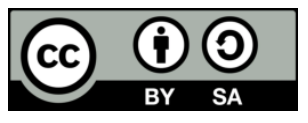

\section{Corresponding Author:}

Lukman Audah

Wireless and Radio Science Centre (WARAS)

Universiti Tun Hussein Onn Malaysia

86400 Parit Raja, Batu Pahat, Johor, Malaysia

Email: hanif@uthm.edu.my

\section{INTRODUCTION}

In this age of communication technology, $5 \mathrm{G}$ technology is becoming a necessary revolution. To bridge the gap between the current internet technology and its ease of use of common man is the challenging task. The most common method to increase the current network capacity with the available speed and frequency range is based on a re-use mechanism for the frequency level [1]. The main goal of internet technology is that the high-speed network in an area of coverage. The partition of the frequency band within the network is possible only with the help of the transfer mechanism [2]. The transfer mechanism is responsible for reallocating the frequency of the current session used by one device to the other [3]. The future use of mobile technology is expected to reach 250 times its current level in 2020. The use of internet of technology for business and real time application reach towards more than 10 billion in 2020. The main objective of the $5 \mathrm{G}$ is the flexibility, scalability, adaptability and realizable network development [4]. To achieve high speed, reliable network researcher is using the software defined network with 5G technology. The triangular problem is faced between the data sharing among the node because of the huge burden on the current network. The cloud computing technology has been currently used for the minimization of weight on 
the current network [5, 6]. For the achieving the capacity for small cell base station HetNets were used towards the development of $5 \mathrm{G}$ dense networks. This network minimize the handover preparation and activation time.

The current mobile networks are witnessing for the ongoing enhancements in traffic and newly connected users [7]. The newly user definitely increases the exponential data traffic among the network. The handover mechanism is a key solution for aim to satisfying the rising requirement in $5 \mathrm{G}$ network $[8,9]$. The uses of the high number of nodes in the networks are creating a challenge towards mobility management. The handover mechanism is working as the partition the frequency band of the available node for the network [10]. The numerous use of handover also makes impact on computer network. The handover needs the delay of time for the functionality. The challenging task is to select the proper handover and implement on proper condition. The channel coding is also a key task for the handover implementation. The performance of the developed network is calculated on the basis of handover delay time [11]. The cellular architecture of 5G using ultra dense network deployment (UND) has been proposed and tested by the researcher. This proposed network has macro base station and radio transmission technology increases 10 times of current frequency. This proposed model will be providing the macro and backbone channel. In this model the optimal small cell base channel (SBS) is connected to substation for the frequency [12]. The average channel capacity is estimated based on spread spectrum and MIMO system. The transmitted signal is extracted bandwidth [13].

Many researchers have been proposed the $5 \mathrm{G}$ handover simulation model for the real time application. The system level simulation and stable performance achievement from the $5 \mathrm{G}$ simulation is discussed by the researcher [14]. The motivated research has been explained in the comparative analysis of $4 \mathrm{G}$ and $5 \mathrm{G}$ simulation network was explained by the researcher [15]. Most of work has been proposed using the open source software MANET routing protocol and simulation [16]. The researcher highlighted different protocol for the testing of the simulation model with respective time parameter [17]. The comparative analysis for commercial and open source simulation model and its results was explained by the researcher $[18,19]$. The researcher are used the ping pong and time based delay handover for the development of reliable 5G network. The description of the handover mechanism is explained in the Figure 1. The Figure 1 explains the received function of receiver frequency from neighbour node of the network. The observation of the neighbour node is transmitted to the base station. The frequency of the neighbour node is transmitted using the downlink manner explained by the researcher [20].

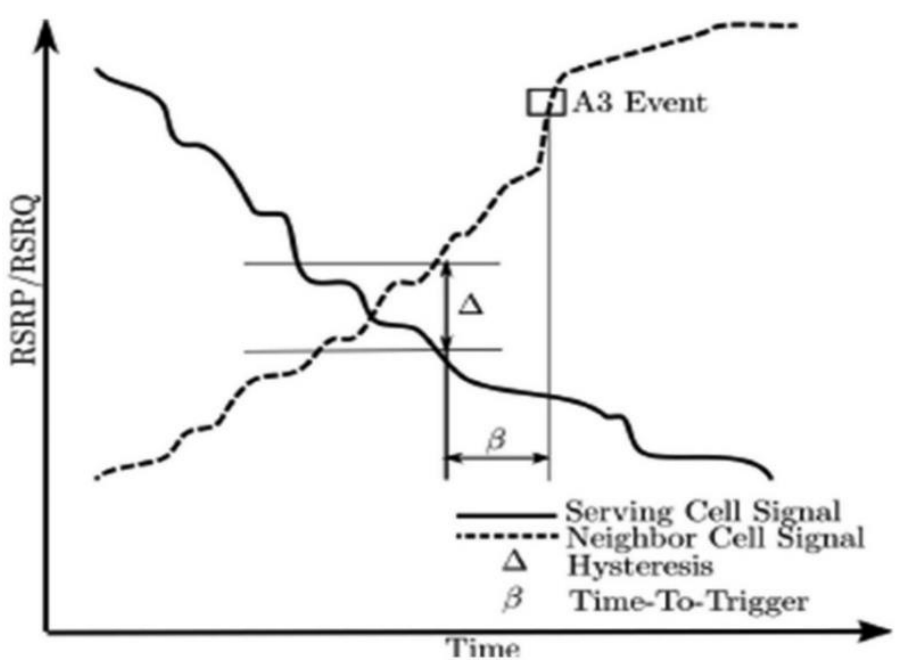

Figure 1. Description of handover mechanism among the cellular network

This research devoted towards design and development of proposed approach of handover based 5G network. The 5G network is design using the intra frequency handover mechanism. In the literature there are various algorithm are used for handover implementation for cellular network [21]. In the proposed network have three levels for the development. The levels are access cloud (AC), regional distributed cloud (RC), and national centralized cloud (NC). From this level we are concentrated on AC and other plane of cloud become unchanged. This research proposed X2 based delay handover for the $5 \mathrm{G}$ network simulator development. The procedure and protocol of LTE standard has been used for this model [22]. In the cellular network frequency of the neighbour node is observing and passed to the base station. The HO implementation and completion 
performance is also highlighted during this research. The researcher has proposed the model using LTE standards and achieves the time as approximately 6.94 from the experimental observation [23]. The traditional handover implementation considers the delay time and serving the frequency partition of the node among converge of the network. From the literature study it is observed that the there is extent need for the design and development of handover based $5 \mathrm{G}$ simulator model. The main objective of this research is to design and implementation of $5 \mathrm{G}$ network based on handover mechanism.

The overall structure of the paper is ordered as given. The section 2 explained proposed research design, and the procedure for the research implementation. The results and discussion regarding the proposed research is described in section 3. The concluding remark followed by the references is included in section 4 .

\section{RESEARCH METHOD}

The technology of $5 \mathrm{G}$ has been set the reliable and trusted network. For the business and IOT application the role of $5 \mathrm{G}$ speed is important [24]. The architecture of the $5 \mathrm{G}$ is combination of the $3 \mathrm{G}$, GPRS and LTE. Each technology has its own server and combination of the server towards the development of $5 \mathrm{G}$ is described in the Figure 2. The $5 \mathrm{G}$ architecture has a main terminal which has an independent and autonomous radio terminal connected using IP link. The packet data is transferred from terminal to 5G terminal.

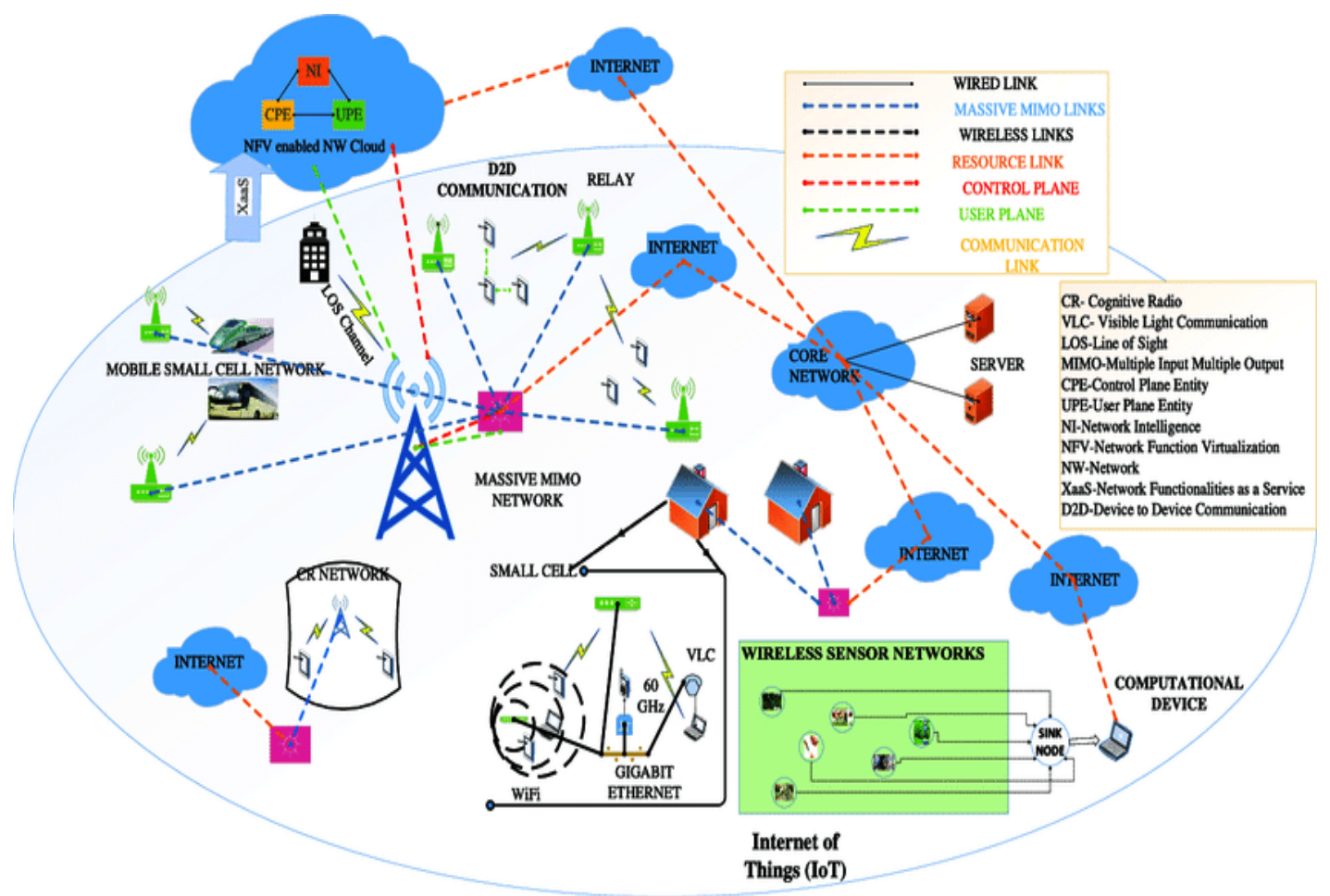

Figure 2. Proposed architecture of 5G technology

The design and development of desirable $5 \mathrm{G}$ network and its real time application setup is the challenging task for the researcher. The simulation model provides the facility to develop the topology, simulated real time executed performance-based model $[25,26]$. On the basis of nodes of network and simulated results provided link between networks the simulation software used by the researcher [27].

For the simulation of the network need the information and target of the network. On the basis of information and target the proposed steps for simulation model is described in Figure 3. From the proposed model the information for the network is collection or gathered. The target for the simulation is set.

Time optimization of the handover mechanism among the macro cell using the velocity of the received signal has been elaborated by the researcher $[28,29]$. The time of the handover optimization in different environment is explained by the various algorithm is explained by the researcher [30, 31]. The most of the researcher are targeted the handover implementation in $5 \mathrm{G}$ network. The base station functionality and 
minimum delay handover implementation is the challenging task. For serving this conditional problem centralized mechanism of handover is implemented through simulation formulation.

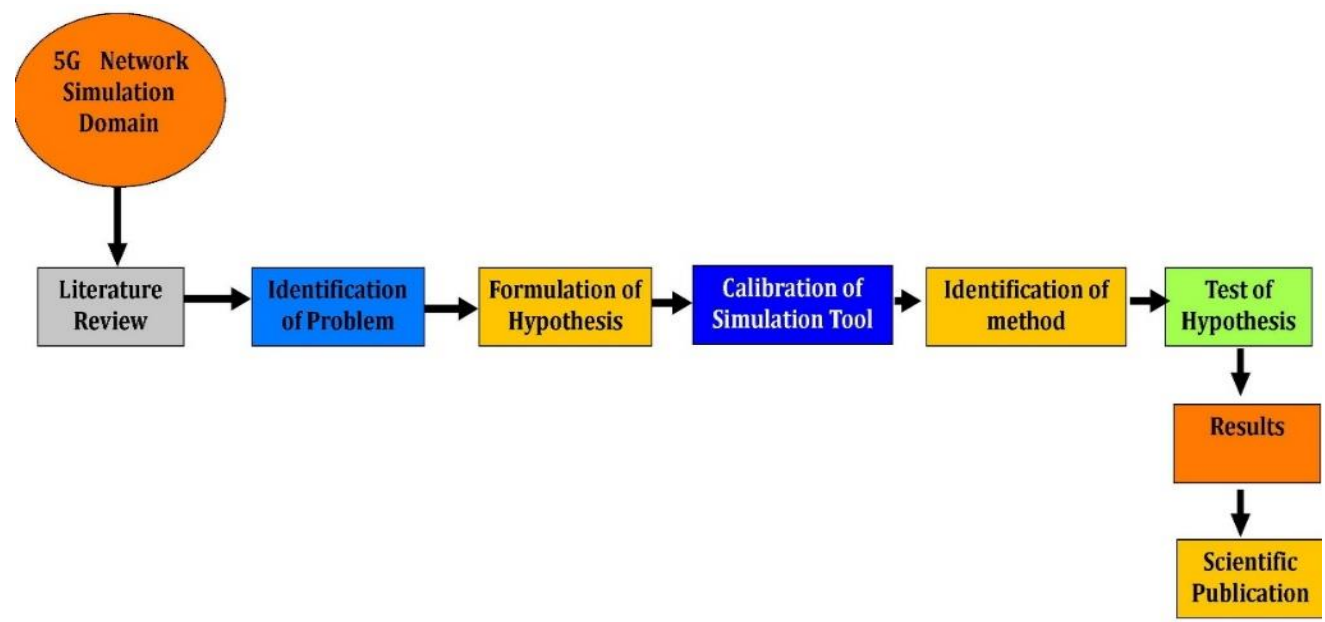

Figure 3. Proposed steps of 5G simulation model

\section{RESULTS AND DISCUSSION}

The experiment is tested for the $5 \mathrm{G}$ simulation model. The Figure 4 describes the graphical representation of the selected parameter for the simulation model. The model is developed for number of sample channel model impulse response. The response is tested on the basis of distance parameter. The performance of the Simulator model is calculated on the basis of time factor. The experiment is tested over the MATLAB simulator on the server system. The features of server include the Intel Xenon $2.40 \mathrm{GHz}$ processor and memory unit of $96 \mathrm{~GB}$.

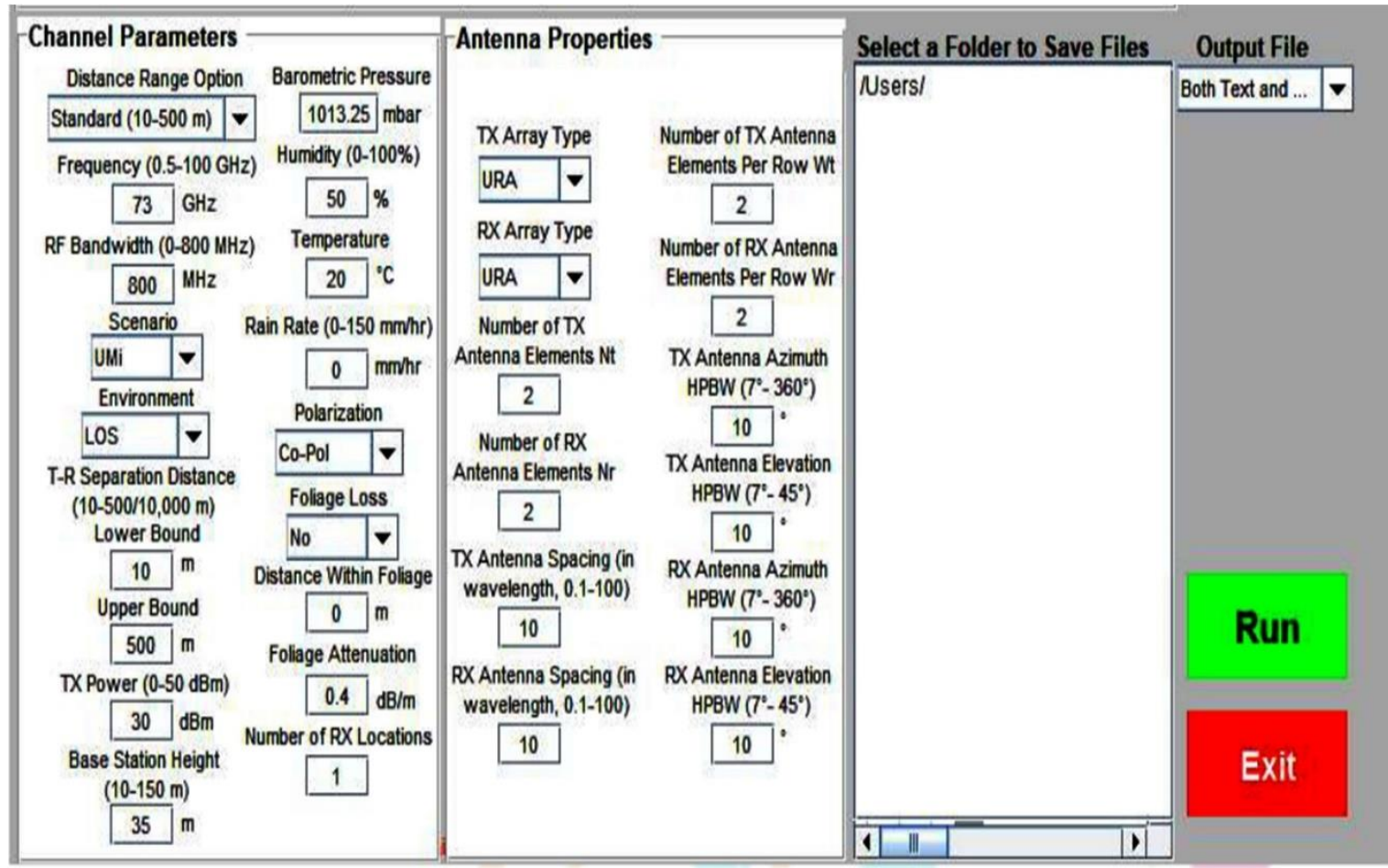

Figure 4. Graphical representation of $5 \mathrm{G}$ simulator model 
From the above graphical representation the total 28 input parameter are passed for the experiment. The 28 parameter are selected from the channel parameter and Antenna properties. The channel has 16 input parameter and 12 parameters are used for the Antenna properties. The architecture of channel parameter used for the simulation experiment is described in Figure 5.

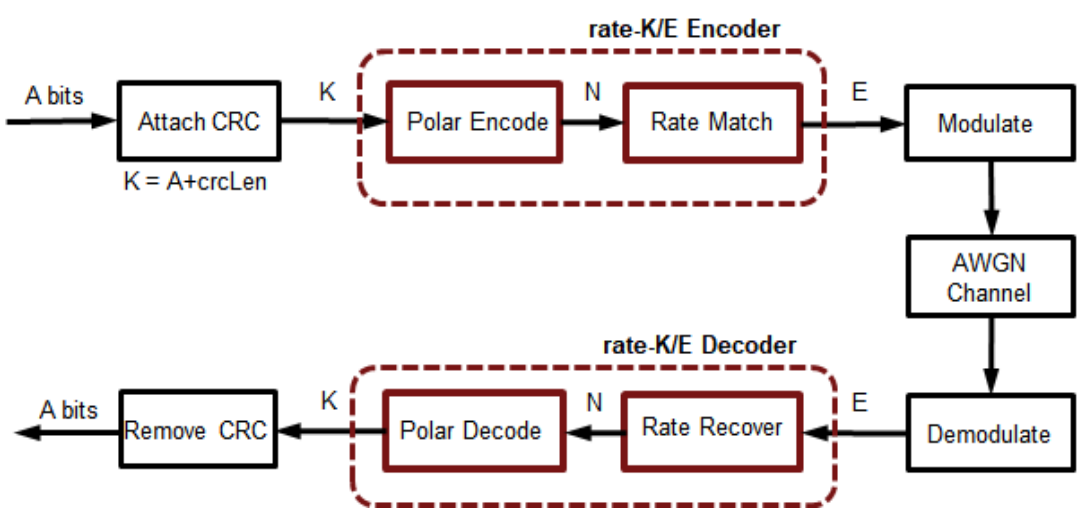

Figure 5. The architecture of channel parameter towards simulation model

The implemented handover implemented for the $5 \mathrm{G}$ network handover network is shown in the Figure 6. The handover is implemented using the SDNC and traffic model. The performance of the simulated network is calculated on the basis of the performance of the simulated network. The topology is the major and challenging task for simulation model. The tested topology for the simulation of the 5G computer network is explained in the Figure 7. The noise level and the channel model for the simulation model are explained in the Figure 8.

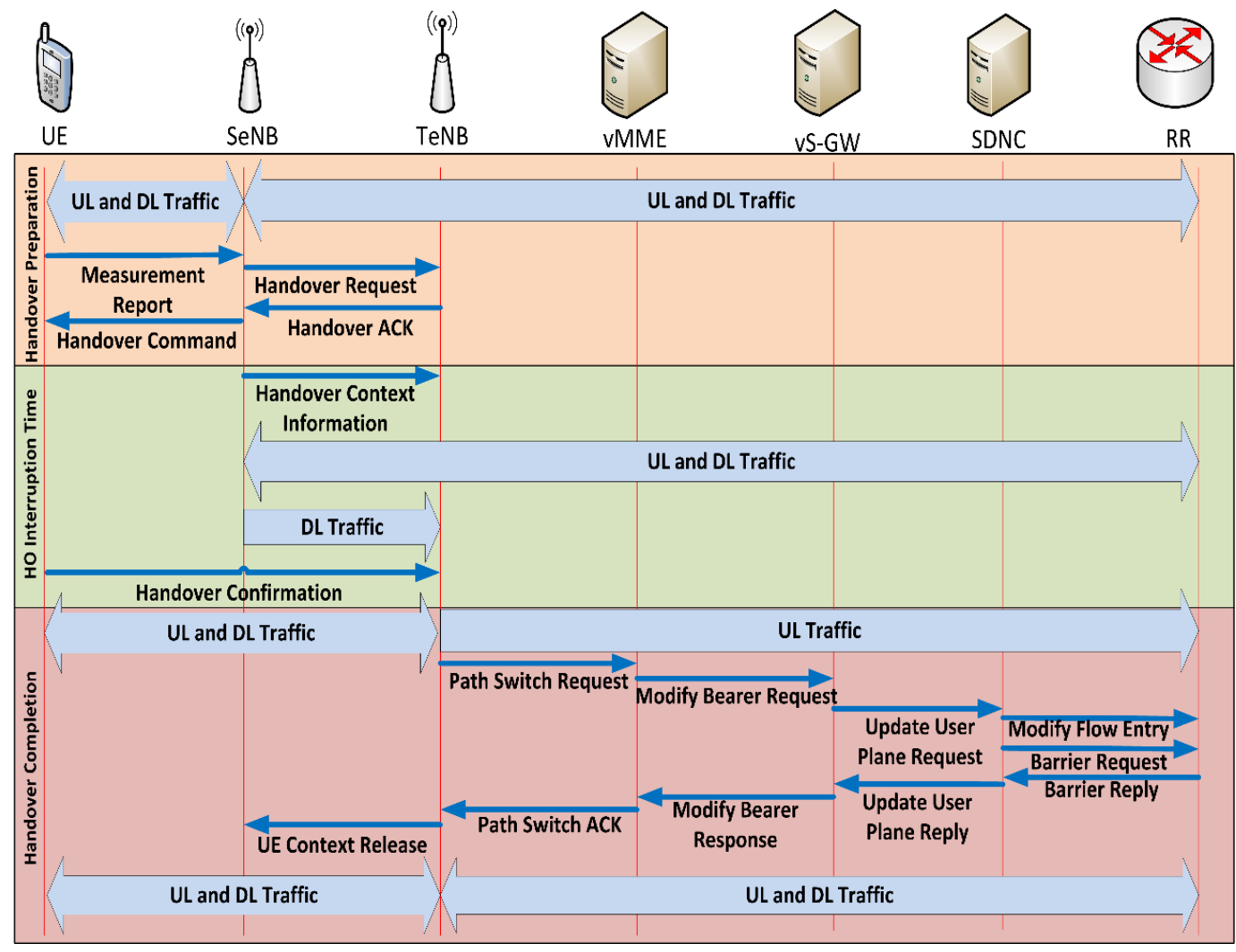

Figure 6. Implemented handover for the 5G simulated network 


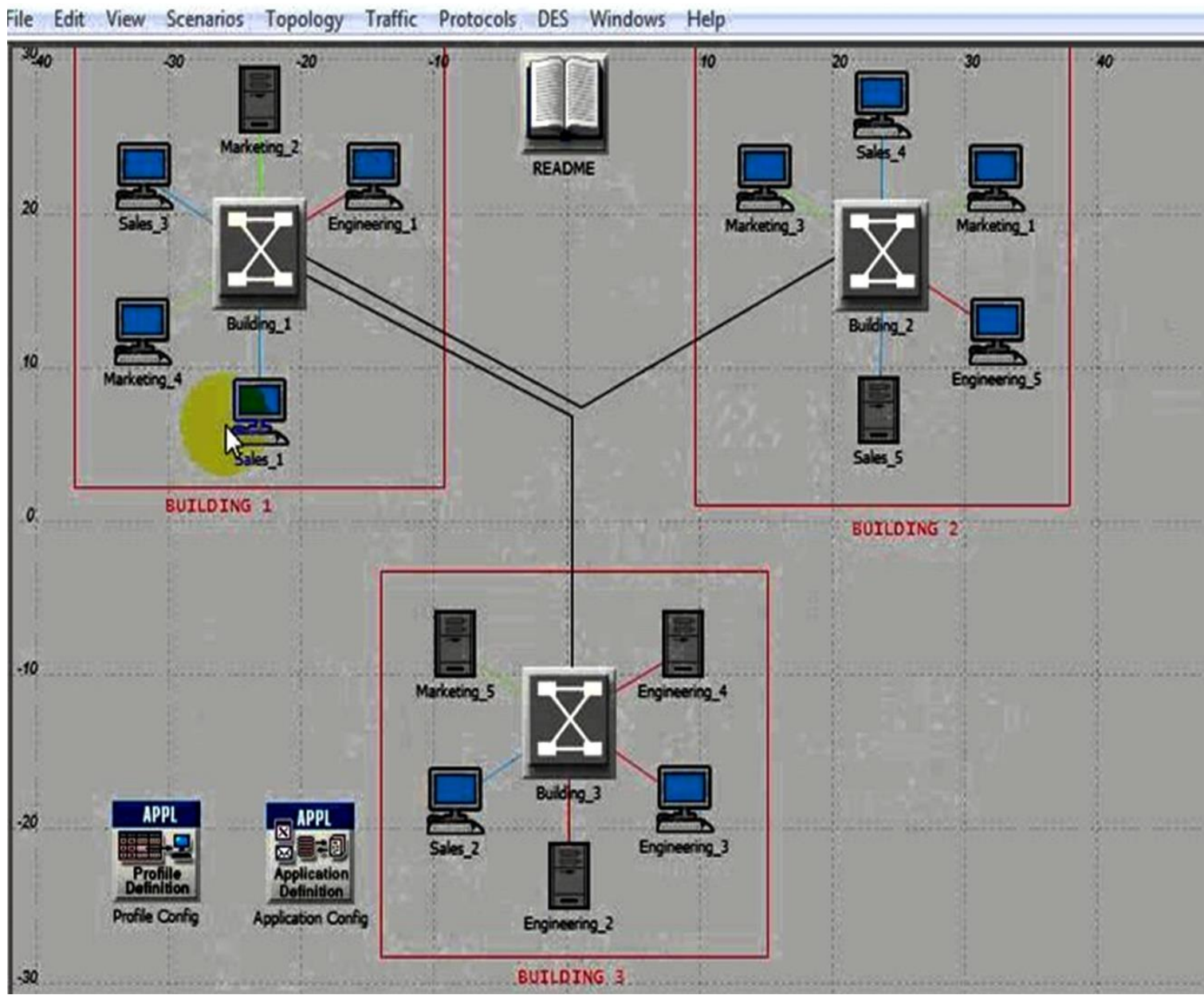

Figure 7. The tested topology for the simulation model

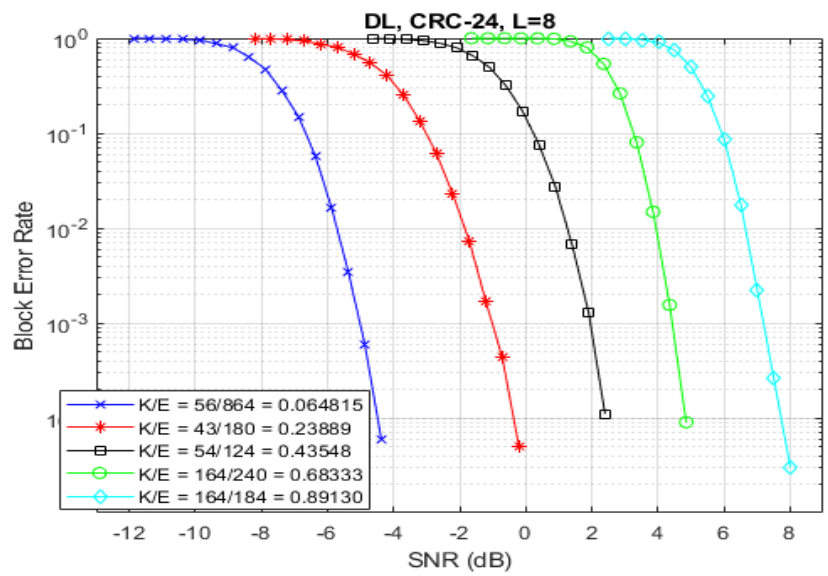

Figure 8. Real time factor with respective to channel specification

The handover implemented and tested using the 5G simulated models. The performance of the handover execution is calculated on the basis of execution time. The graphical representation for average rate and number of user is explained in Figure 9. The Figure 9 explained the representation of average rate and number of user for the transmission in the signal. From Figure 9, it observed that as increase data rate number of user is also increases. The average rate and number of base station is represented in Figure 10. 


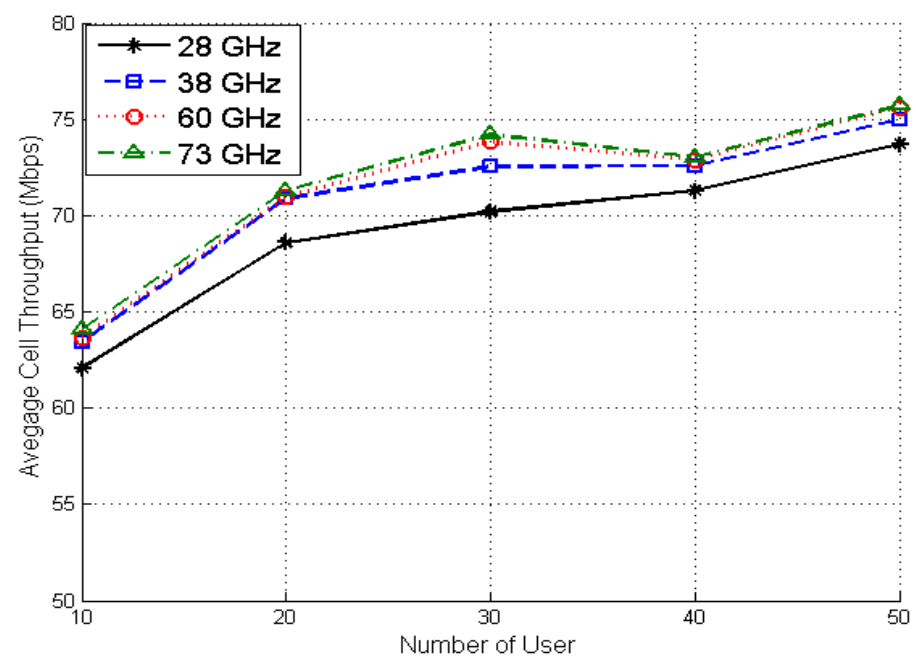

Figure 9. Average rate vs number of user

The variation based representation for average rate vs number of base station such as 10, 20, 30, 40 and 50 is explained in Figure 10. From Figure 10, it is observed that the base station increases then the data rate become decrease. The graphical representation of the handover preparation and extraction time is explained in Figure 11. From Figure 11, observed that the handover execution time is the sum of these contributions and the HO interruption time. The graphical representation of the data rate and delay for the handover execution time is extracted. We assumed a constant handover interruption time of $15 \mathrm{~ms}$ and observed that HO preparation and completion times are almost constant for data traffic rates per UE up to 1 Gbps. From this point on the HO, execution time increases because the backhaul network begins to exhibit congestion. In other words, the time spent waiting in queues at BN rise. Handover interrupts time is directly affecting the performance of the simulation network. The delay of the transmission increases, it directly increases the failure rate of handoff in the transmission.

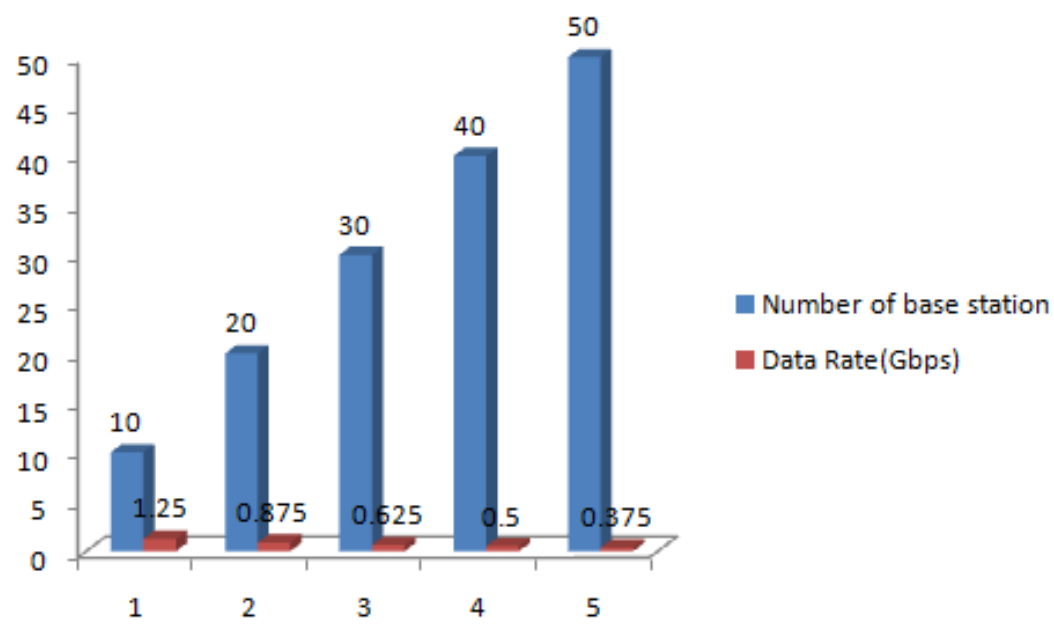

Figure 10. Average rate vs number of base station

From the literature experiment it is observed that the delay of handover and handover preparation is the $8.0 \mathrm{~ms}$ and handover completion time is $10.78 \mathrm{~ms}[11,15]$. The proposed handover from the researcher is used for the coverage planning and $5 \mathrm{G}$ development. From the above literature results it is observed that the extracted handover preparation time like $7.08 \mathrm{~ms}$ and handover completion time is $9.98 \mathrm{~ms}$ is optimal. The delay of the handover is $2.9 \mathrm{~ms}$. The performance of the proposed $5 \mathrm{G}$ is extreme level because the delay time is optimal. 


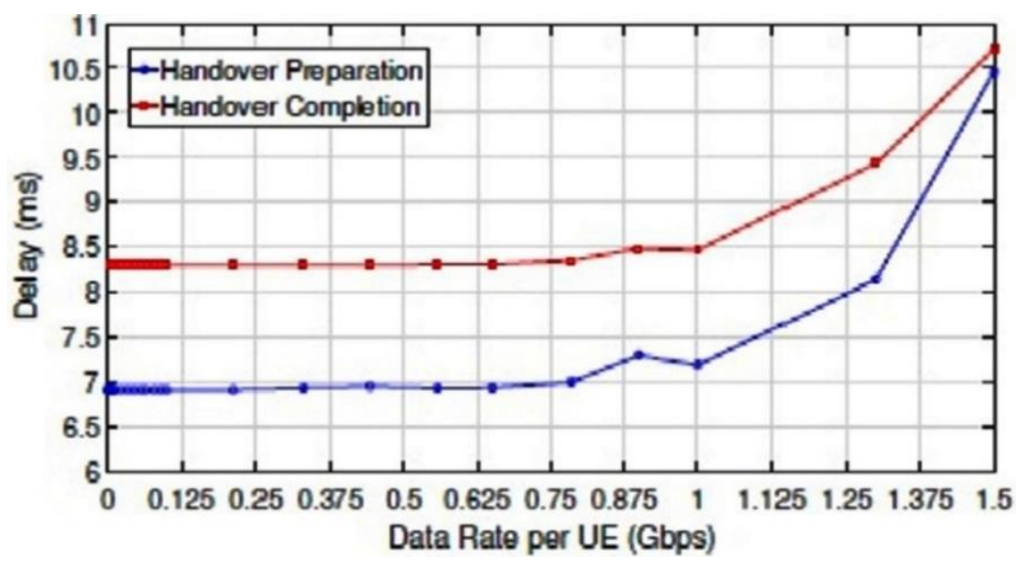

Figure 11. Performance of the handover simulation model on the basis of execution time

\section{CONCLUSION}

In this research the design and development of $5 \mathrm{G}$ handover implementation has been carried out. In this experiment the MATLAB software are used for the simulation network development. The channel model and Antenna mode are used for the development. The 28 parameters are passed for the experimental analysis in which 16 are for channel parameter. Twelve parameters are used for the Antenna properties. The system level simulator model is developed from this experiment. From the simulator we have developed the topology and handover implementation. The simulator is tested over the frequency range of $500 \mathrm{MHz}$ to $150 \mathrm{GHz}$ and radio frequency for the $700 \mathrm{MHz}$ bandwidth. The design model is compared with the $3 \mathrm{G}$ and 4G simulator model. The performance of the simulation system is calculated on the basis of handover preparation and completion time. This experiment achieves the $7.08 \mathrm{~ms}$ handover preparation time and 9.98 $\mathrm{ms}$ handover completion time. The delay time for the handover is $2.9 \mathrm{~ms}$. The author recommended that to perform the future work regarding to minimize the delay of to improve the performance of the $5 \mathrm{G}$ cellular technology.

\section{REFERENCES}

[1] Gharsallah, Amina, Zarai, "MIH/SDN-Based Vertical Handover Approach For 5G Mobile Networks," Journal of Information Science and Engineering,vol. 35, no. 5, pp. 1161-1172, Sep. 2019.

[2] X. Jiangwei et al., "Method and apparatus for handover in mobile content centric network," US Patent, Jan. 2018.

[3] G. Liu and D. Jiang, "5G: Vision and requirements for mobile communication system towards year 2020," Chinese Journal of Engineering, 2016.

[4] P. Tayyaba, S. Khan, and M. A. Shah, "5G cellular network integration with SDN: Challenges, issues and beyond," 2017 International Conference on Communication, Computing and Digital Systems (C-CODE), 2017, doi: 10.1109/C-CODE.2017.7918900.

[5] Costa-Requena, J., Santos, J. L., Guasch, V. F., Ahokas, K., Premsankar, G., Luukkainen, S. et al., "SDN and NFV integration in generalized mobile network architecture," in 2015 European Conf. on. IEEE Networks and Commun. (EuCNC), 2015, pp. 154-158, doi: 10.1109/EuCNC.2015.7194059.

[6] M. Dighriri, G. M. Lee, and T. Baker, "Applying scheduling mechanisms over 5G cellular network packets traffic," Third International Congress on Information and Communication Technology, Singapore, 2019, doi: 10.1007/978-981-13-1165-9_11.

[7] Vasudeva, K., Dikmese, S., Güvenç, I., Mehbodniya, A., Saad, W., and Adachi, F., "Fuzzy logic game-theoretic approach for energy efficient operation in hetnets," 2017 IEEE International Conference on Communications Workshops (ICC Workshops), 2017, doi: 10.1109/ICCW.2017.7962716.

[8] K. Alghamdi and R. Braun, "Software defined network (SDN) and OpenFlow protocol in 5G network," Communications and Network, vol. 12, no. 01, 2020, Art. no. 28.

[9] M. Joud, M. García-Lozano, and S. Ruiz, "User specific cell clustering to improve mobility robustness in 5G ultradense cellular networks," in Proceeding of 14th Annual Conference on Wireless On-demand Network Systems and Services (WONS), Isola, 2018, pp. 45-50.

[10] Lu, Y., Zhang, M., Song, C., Guan, L., Wang, D., Li, S., and Zhan, Y., "A Multi-Migration Seamless Handover Scheme for Vehicular Networks in Fog-based 5G Optical Front haul," 24th Optoelectronics and Communications Conference (OECC) and 2019 International Conference on Photonics in Switching and Computing (PSC), Fukuoka, Japan, 2019, pp.1-3, doi: 10.23919/PS.2019.8817683.

[11] M. Tayyab, X. Gelabert, and R. Jäntti, "A Simulation Study on Handover in LTE Ultra-Small Cell Deployment: A 5G Challenge," IEEE 2nd 5G World Forum (5GWF), Dresden, Germany, 2019, pp. 388-392. doi: 10.1109/5GWF.2019.8911616. 
[12] Abbas, K., Afaq, M., Ahmed Khan, T., Rafiq, A., Iqbal, J., Ul Islam, I., and Song, W. C., “An efficient SDN-based LTE-WiFi spectrum aggregation system for heterogeneous 5G networks," Transactions on Emerging Telecommunications Technologies, 2020, doi: 10.1002/ett.3943.

[13] P. Varzakas, "Average channel capacity for Rayleigh fading spread spectrum MIMO systems," International Journal of Communication Systems, vol. 19, no. 10, pp. 1081-1087, 2006.

[14] Campanile, L., Gribaudo, M., Iacono, M., Marulli, F., and Mastroianni, M., "Computer network simulation with ns3: A systematic literature review," Electronics, vol. 9, no. 2, 2020, Art. no. 272.

[15] X. Zhou and H. Tian, "Comparison on network simulation techniques," in 17th International Conference on Parallel and Distributed omputing, Applications and Technologies (PDCAT), Dec. 2016, pp. 313-316.

[16] O. Semenova, A. Semenov, and O. Voitsekhovska, "Neuro-Fuzzy Controller for Handover Operation in 5G Heterogeneous Networks," 3rd International Conference on Advanced Information and Communications Technologies (AICT), Lviv, Ukraine, 2019, pp. 382-386.

[17] Q. Liu, C. F. Kwong, S. Zhang et al., "A fuzzy-clustering based approach for MADM handover in 5G ultra-dense networks," Wireless Network, 2019.

[18] P. K. Gkonis, P. T. Trakadas, and D. I. Kaklamani, "A Comprehensive Study on Simulation Techniques for 5G Networks: State of the Art Results, Analysis, and Future Challenges," Electronics, vol. 9, no. 3, 2020, Art. no. 468, doi: 10.3390/electronics9030468.

[19] V. Yajnanarayana, H. Ryd'en, L. 'o H'evizi, A. Jauhari, and M. Cirkic, "5G Handover using Reinforcement Learning," arXiv:1904.02572v2, Apr. 2019.

[20] Lai, W. K., Shieh, C. S., Chou, F. S., Hsu, C. Y., and Shen, M. H, "Handover Management for D2D Communication in 5G Networks," Applied Sciences, vol. 10, no. 12, 2020, Art. no. 4409.

[21] P. Sapkale and U. Kolekar, "Handover Decision Algorithm for Next Generation," Proceedings of International Conference on Wireless Communication, Singapore, vol. 10, no. 12, 2020, Art. no. 4409, doi: 10.3390/app10124409.

[22] A. Kaloxylos, "A survey and an analysis of network slicing in 5G Networks," IEEE Commun. Stand. Mag., vol. 2 ,no. 1 , pp. $60-65,2018$.

[23] E. Hamza and S. Nafea, "Design and Analysis WIMAX Network Based on Coverage Planning," Journal of Engineering, vol. 26, no. 2, pp. 99-110, 2020. doi: 10.31026/j.eng.2020.02.08.

[24] Lavacca, F. G., Salvo, P., Ferranti, L., Speranza, A., and Costantini, L., "Performance Evaluation of 5G Access Technologies and SDN Transport Network on an NS3 Simulator," Computers, vol. 9, no. 2, 2020, Art. no. 43 doi: 10.3390/computers9020043.

[25] Ouali, K., Kassar, M., Nguyen, T. M. T., Sethom, K., and Kervella, B., “An Efficient D2D Handover Management Scheme for SDN-based 5G networks," 2020 IEEE 17th Annual Consumer Communications \& Networking Conference (CCNC), 2020, pp. 1-6.

[26] N. Aljeri and A. Boukerche, "Mobility Management in 5G-enabled Vehicular Networks: Models, Protocols, and Classification," ACM Computing Surveys (CSUR), vol. 53, no. 5, pp. 1-35, 2020.

[27] G. Borboruah and G. Nandi, "A study on large scale network simulators," International Journal of Computer Science and Information Technologies, vol. 5, no. 6, pp. 7318-7322, 2014.

[28] A. Yazdinejad, R. M. Parizi, A. Dehghantanha, and K. R. Choo, "Blockchain-enabled Authentication Handover with Efficient Privacy Protection in SDN-based 5G Networks," in IEEE Transactions on Network Science and Engineering, 2019, doi: 10.1109/TNSE.2019.2937481.

[29] M. Samra, L. Chen, C. Roberts, C. Constantinou, and A. Shukla, "TV White Spaces Handover Scheme for Enabling Unattended Track Geometry Monitoring From In-Service Trains," in IEEE Transactions on Intelligent Transportation Systems, 2020, pp. 1161-1173.

[30] A. N. Kasim, "A Survey Mobility Management in 5G Networks," arXiv:2006.15598, 2020.

[31] K. Ahmadi, S. P. Miralavy, and M. Ghassemian, "Software-defined networking to improve handover in mobile edge networks," International Journal of Communication Systems, vol. 33, no. 14, 2020, doi: 10.1002/dac.4510. 